\title{
Characteristics of piano sound (Acoustical research on the piano, Part 4)
}

\author{
Isao Nakamura \\ Faculty of Informatics, Teikyo Heisei University, \\ 2289-23 Ohtani, Uruido, Ichihara, 290-01 Japan
}

(Received 22 March 1996)

【English translation of the same article in J. Acoust. Soc. Jpn. (J) 38, 454-460 (1982)】

\begin{abstract}
This paper investigates the characteristics of piano sound using the waveforms of velocity of vibration of strings, the waveforms of driving force and velocity of vibration of the soundboard, and waveforms of sound pressure. The waveforms for the investigation were generated by using a analog simulator so that the separation of conditions is easy. A pulse generated by striking a string with a hammer propagates along the string and reflects against the soundboard. This drives the soundboard producing its vibration, and a part of vibration energy radiates from the soundboard producing piano sounds. The soundboard vibration contains both forced and free vibrations. The latter is influenced by the driving point admittance of the soundboard, and this plays an important role in determining the piano timbre. Still more the characteristics of piano sound are clarified using waveforms obtained by digital simulation and their spectra.
\end{abstract}

Keywords: Waveform, Formation of piano sound, Analog simulator, Digital simulation, Piano timbre

PACS number: 43. 75. Mn, 43. 75. $\mathrm{Wx}, 43.58 . \mathrm{Ta}$

\section{INTRODUCTION}

Piano sound ranges from $27.5 \mathrm{~Hz}$ (fundamental frequency of key number $1, \mathrm{~A} 0$ ) to $4,186 \mathrm{~Hz}$ (fundamental frequency of key number $88, \mathrm{C} 8$ ), and they are all in transient states. The sounds are produced by a complex vibration system consisting of hammers, strings, and the soundboard with bridges and ribs. It is difficult to understand the mechanism of the sound by directly analysing actual sounds. This series of studies aims at finding the characteristics of the piano sound by analysing its sound generation mechanism; i.e. the vibrations of strings struck by a hammer, ${ }^{1)}$ the vibrations and acoustic characteristics of the soundboard in a steady state, ${ }^{2)}$ coupling conditions of strings and the soundboard, and time variations of piano sound with strings. ${ }^{3)}$ Based on the results of the previous papers, this paper describes the fundamental patterns of the piano sound.

In 1958, J. P. Quitter ${ }^{4)}$ investigated the characteristics of piano sound to design an electric piano which uses vibrations of reeds. In 1969, P. R. Dijksterhuis and T. Verhey ${ }^{5)}$ investigated the piano sound to design their electronic piano. The characteristics obtained by both the studies were approximate. In 1943, O. H. Schuck and R. W. Young ${ }^{6}$ made an analytical study in which they found that partials in a higher frequency dacay faster than those in a lower frequency. In 1962, H. Fletcher et al. ${ }^{7}$ found that the inharmonicity caused by the elasticity of strings is a characteristic of the piano sound. The twostage attenuation of the piano sound had been pointed out by D. W. Martin. ${ }^{8)}$ After that, G. Weinreich, ${ }^{9)}$ T.C. Hundley et al., ${ }^{10)}$ and $\mathrm{I}$. Nakamura $^{3)}$ clarified respectively that the two different attenuation rates in each note are due to multiple strings. H. Fletcher et al. ${ }^{7)}$ did not men- 
tion the mechanism of generation of partials which produce the characteristic sound of the piano.

This paper investigates the mechanism of spectrum of each piano note quantitatively, notably the relationship between lower note strings and the soundboard, so that the basic characteristics of the piano sound are represented by physical constants.

\section{WAVEFORMS OF VIBRATIONS AND SOUND OF THE PIANO}

Although J. P. Quitter ${ }^{4)}$ and R. D. Weyer ${ }^{11}$ have shown waveforms of the piano sound in their papers, they have not specified the measurement conditions. The waveforms in their paper appear to be greatly influenced by the positions of the measurements. In this paper, the waveforms of vibrations and sound pressure of an upright piano were measured at various points which are relevant to the sound generation process. Figure 1 shows typical oscillograms of sound generated by a single, double and triple string; key number 5 ( $\mathrm{Cl \# ,} 34.6$ $\mathrm{Hz})$, key number $15(\mathrm{~B} 1,61.7 \mathrm{~Hz})$, and key number 30 (D3, $146.8 \mathrm{~Hz})$ respectively. (a), (b) or (c) shows the velocity waveform of each string measured by a magnetic pick-up at the central symmetry position to the string struck point. $\left(a^{\prime}\right),\left(b^{\prime}\right)$ or $\left(c^{\prime}\right)$ shows the waveform of the driving-force at each supporting position of the string on the bridge. $\left(a^{\prime \prime}\right),\left(b^{\prime \prime}\right)$ or $\left(c^{\prime \prime}\right)$ shows the waveform of the vibration velocity of the soundboard at the same position as in $\left(a^{\prime}\right),\left(b^{\prime}\right)$ or $\left(c^{\prime}\right)$.

All the waveforms of the driving forces of the soundboard indicate the propagation of a pulse along each string. The pulse consists of two halfsine waves, one being positive and the other one negative. ${ }^{1)}$ The higher the wave frequency, the faster the wave propagation, due to the elasticity of the string. Therefore, each waveform changes with time due to the dispersion. In Fig. $1\left(a^{\prime}\right)$, a singlehead arrow indicates a preceding wave, and a double-head arrow a delaying wave.

Although the soundboard is driven by the force of each string, its waveform shows a mixed free vibration. This is a very important fact in determining the piano timbre. Note, Nakamura ${ }^{12)}$ is the only worker who pointed out this fact. Since the piano sound is influenced by the driving point admittance of the soundboard, the sound with the soundboard is completely different from that produced by strings alone.

Figure 2 shows the method of obtaining the waveform of the driving force of the soundboard from the waveform of its velocity. Theoretically, the waveform of the velocity of a string is given by a superposition of the waveform of the vertical tension and its reflected wave. ${ }^{1)}$ The former is the vertical component of the tension of the string. The latter has a time lag by $2 a$, and its phase is reversed; where $a$ is the distance between the position of a hammer on the string and the fixed end of the string. Therefore, the waveform of the vertical force component of the string is obtained by feeding back the waveform of the velocity of the string through a delay circuit having delay time of $2 a / c_{0}$,

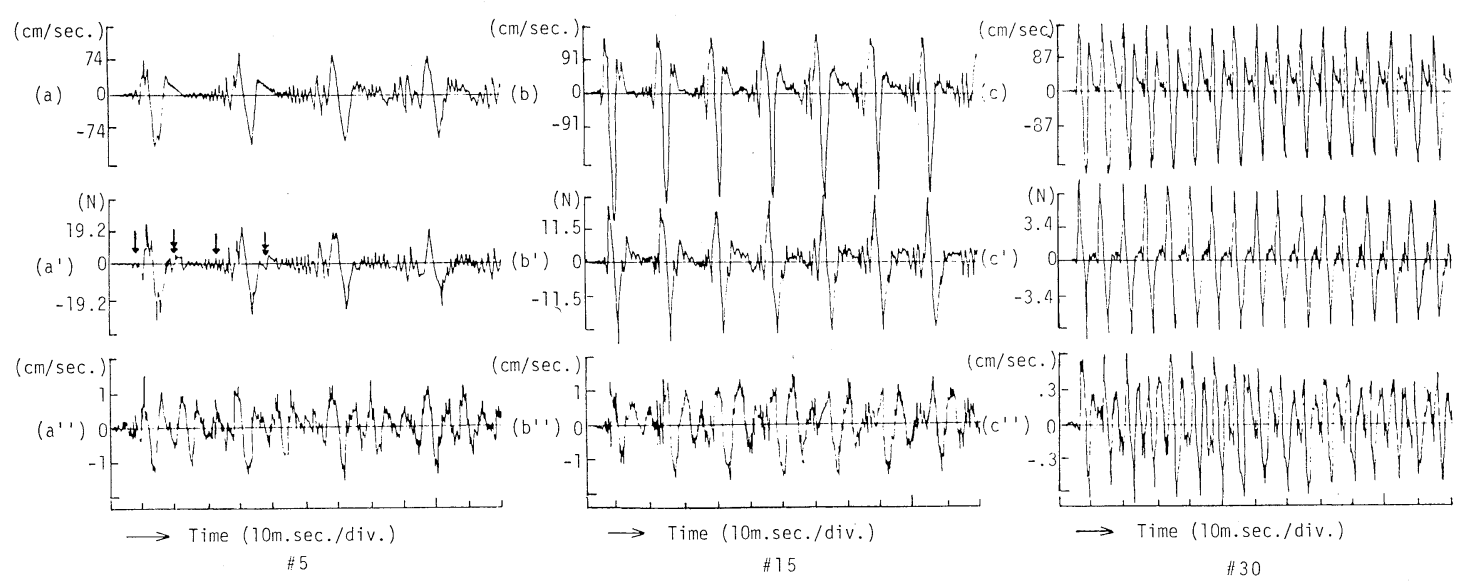

Fig. 1 Waveforms of vibrations of strings and soundboard, measured. (a)-(c): Velocity of string vibration. $\left(a^{\prime}\right)-\left(c^{\prime}\right)$ : Diving force at bridge on soundboard. $\left(a^{\prime \prime}\right)-\left(c^{\prime \prime}\right)$ : Velocity of soundboard at driving point. 


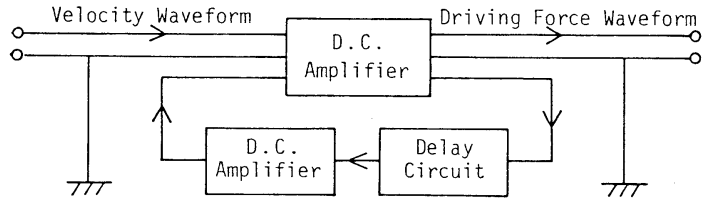

Fig. 2 Electronic system to obtain driving force waveform from velocity waveform.

$c_{0}$ being the propagation velocity. This positive feed back with appropriate coefficient produces a waveform of

$$
\begin{gathered}
e(t)-e\left(t-\frac{2 a}{c_{0}}\right) \mathbf{1}\left(t-\frac{2 a}{c_{0}}\right) \\
+e\left(t-\frac{2 a}{c_{0}}\right) \mathbf{1}\left(t-\frac{2 a}{c_{0}}\right)-e\left(t-\frac{4 a}{c_{0}}\right) \mathbf{1}\left(t-\frac{4 a}{c_{0}}\right) \\
+e\left(t-\frac{4 a}{c_{0}}\right) \mathbf{1}\left(t-\frac{4 a}{c_{0}}\right) \\
-\cdots=e(t) .
\end{gathered}
$$

Figure 3 shows the waveforms of the vibration velocity of the soundboard and its sound pressure measured simultaneously. In these measurements, each string shown in Fig. 1 was struck with an intensity double or triple the force used to obtain waveform in Fig. 1. The measurements were carried out in a sound-proof room with walls covered by rockwool, glasswool and velvet (not perfectly anechoic). The sound pressure was measured at a position distant from the centre of the soundboard by $3.8 \mathrm{~m}$. Since the measuring point is near a wall of the room, lower sounds contain slight reflections from the wall. The waveforms of the sounds measured show a similarity to the those of vibrations of the soundboard, although not exactly the same. This is due to sound pressure radiating from a large area of the soundboard with a low efficiency in a low sound range, and some reflections from room walls. Nevertheless, the measurements clearly demonstrate the point that a pulse-like forced vibration with a period of pitch and a free vibration of the soundboard occur simultaneously. This type of compound vibration is not clearly seen in a very low sound range. The amplitude of the piano sound is near the maximum at its first peak, as in the waveform of vibration of the soundboard.

Obtaining the waveform of sound pressure from that of vibration of the soundboard means a consideration of the transmission characteristic of the whole piano including its case. However, the sound of a piano set in an anechoic room is not good due to the lack of sound reflection. Therefore, analysis of the piano sound in this paper is limited to the vibration velocity of bridges on the soundboard, leaving a discussion of piano timbre to another occasion.

\section{FORMATION OF PIANO SOUND USING SOUNDBOARD}

The impedance of the soundboard has been discussed in the previous paper. ${ }^{2)}$ Figures 4(a) and 4(b) show the frequency responses of the drivingpoint admittances of key numbers 5 and 15 respectively. Figure $4\left(a^{\prime}\right)$ and $\left(b^{\prime}\right)$ show the frequency responses of driving-point reactances of key numbers 5 and 15 respectively, together with their smoothed curves and the positions of the partial frequencies, which were obtained from their admittances and phase characteristics. Their resistance characteristics were also obtained at the same time (not shown). The position of the driving point in these key numbers is on the bass bridge for the near edge drive of the soundboard, and its resonant fre-

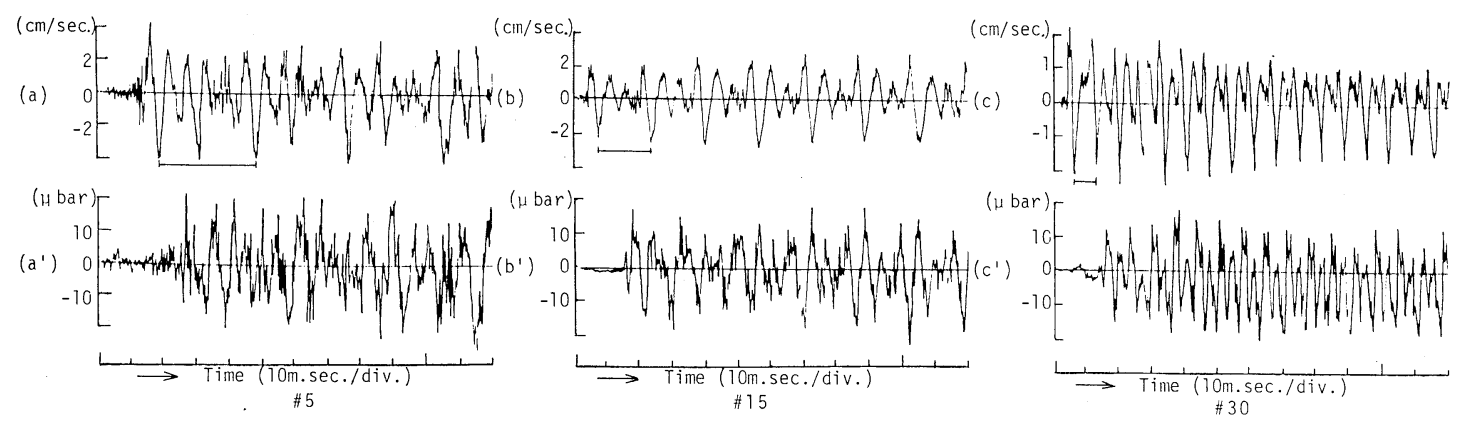

Fig. 3 Waveforms of vibration of soundboard and their sounds, measured. (a)-(c) : Velocity of soundboard at driving point. $\left(\mathrm{a}^{\prime}\right)-\left(\mathrm{c}^{\prime}\right)$ : Sound pressure at a distance from soundboard of $3.8 \mathrm{~m}$. 

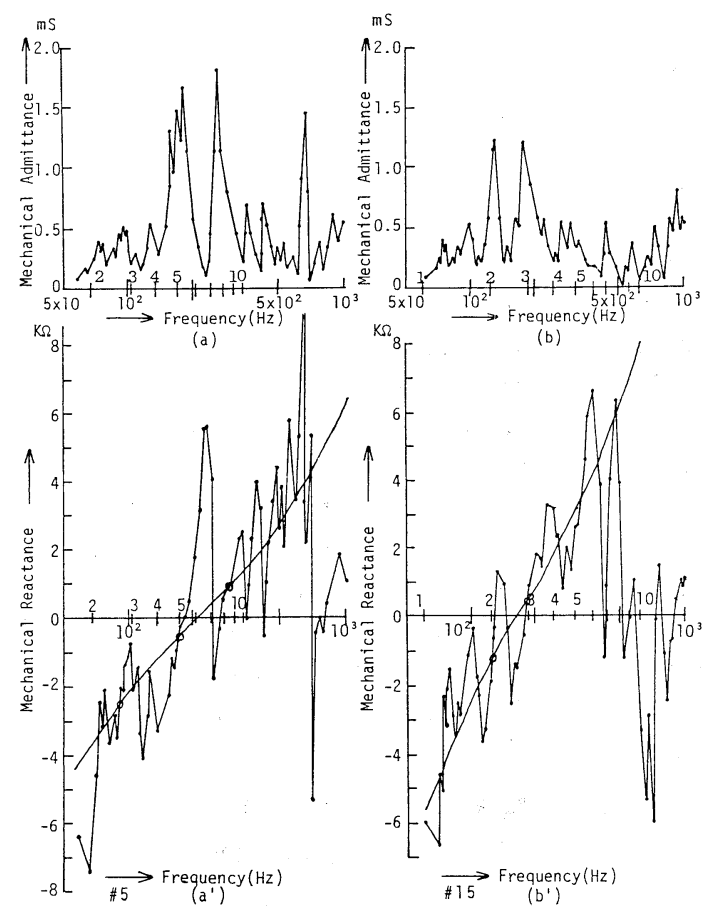

Fig. 4 Frequency responses of driving-point admittance and reactance.

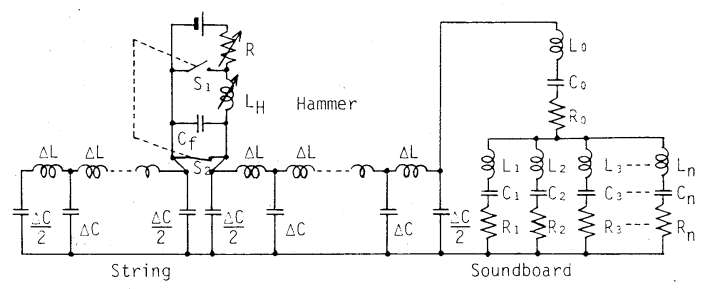

Fig. 5 Analog simulator for piano system (simplified illustration).

quency is about $200 \mathrm{~Hz}$. At the right part of Fig. 5, the impedance of the soundboard in a low frequency sound is represented by $L_{0}, C_{0}$ (corresponding to a smoothed curve in Fig. 4), their resistance component being $R_{0}$, and other elements being from $L_{1}, C_{1}$, $R_{1}$ to $L_{n}, C_{n}, R_{n}$ (each correspond to details of the vibration mode). Figure 5 shows a simplified analogue simulator (modified from the simulator in the previous paper ${ }^{1)}$ of a piano string and hammer system with the driving-point impedance of the soundboard. The string in the simulator consists of 60 meshes. $L_{\mathrm{H}}$ represents the mass of a hammer, $C_{\mathrm{r}}$ represents the compliance of hammer felt. The simulator has two switches; $S_{1}$ and $S_{2}$. When $S_{1}$ is off and $\mathrm{S}_{2}$ is on, they represent the hammer separated from the string. When these switches are reversed, they represent the hammer on the string. The switches are controlled automatically by a transistor circuit synchronised with a pulse generator. The electrical system in the simulator was transformed from the mechanical system in a piano, with appropriate coefficients for time and impedances. The simulator has only one simulated string, but this is enough for an analysis of initial state of string vibration. A string and the soundboard cannot accurately be simulated by an electrical system. Notably, an attenuation of vibration of a simulated string is faster than the real string, since the $Q$ of the inductors used are not high enough. However, this simulator using variable inductors has an advantage that most waveforms can be reproduced simply.

Figures 6(a) and 6(b) show the waveforms of the driving force of the soundboard at key numbers 5 and 15 respectively. Figures $6\left(a^{\prime}\right)$ and $6\left(b^{\prime}\right)$ show those of velocity waves. The units of the vertical axes of the figures are given by using electrical terms for convenience, but the waveforms alone are the subject of the discussion. To simulate the driving impedance at key number 5 , three series resonance elements in a parallel connection plus one resonance element in a series connection were used. Each of the three resonance elements has a frequency indicated by a circle in Fig. 4. To simulate the driving force at key number 15 , two resonance elements in a parallel connection plus one series resonance element in series connection were used. These arrangements are a simplified representation of the vibration system around a part where the frequency characteristic curve of the synthesized reactance crosses the zero level. However, the $Q$ factor of the elements cannot accurately be represented. The propagation velocity of a wave becomes slower at a higher frequency in the electrical system, because of its quantized representation of the string. In fact, this becomes faster at a higher frequency in the actual piano system, because of the elasticity of a string. Therefore, the degree of the simulation deteriorates at a higher frequency. Nevertheless, the simulated waveforms shown in Figs. $6\left(a^{\prime}\right)$ and $6\left(b^{\prime}\right)$ are similar enough to the measured waveforms from the piano shown in Fig. 1(a") and Fig. 3(a); and Fig. 1( $\left(b^{\prime \prime}\right)$ and Fig. 3(b) respectively. All these results show that a free vibration of the soundboard 


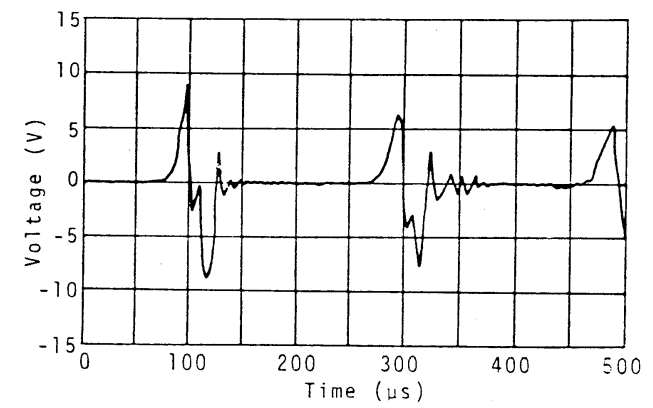

(a)

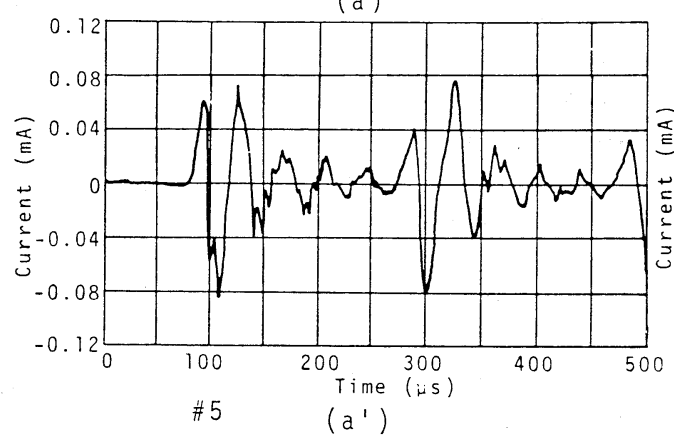

Fig. 6 Waveforms of vibration of soundboard, obtained by simulator. (a), (b) : Driving force of soundboard. $\left(a^{\prime}\right),\left(b^{\prime}\right)$ : Velocity of soundboard at driving point.

is due to the driving point impedances.

Let us consider a case where the driving-point impedance of the soundboard consists of a single series resonance system, and a pulse generated on a string by a hammer does not come back during the time when the hammer is on the string. Then the vibration velocity of the soundboard is given by

$$
\begin{aligned}
I= & \frac{i_{0}}{2} \frac{\sqrt{\omega_{\mathrm{f}} L_{\mathrm{s}} D}}{\omega}\left\{\frac{\alpha^{2}}{\omega} e^{-\alpha t} \sin \left(\omega t+\theta_{1}\right)\right. \\
& \left.+\frac{\sqrt{\omega_{\mathrm{s}}{ }^{2}+\alpha_{\mathrm{s}}{ }^{2}}}{\omega_{\mathrm{s}}} e^{-\alpha_{\mathrm{s}} t} \sin \left(\omega_{\mathrm{s}} t+\theta_{2}\right)\right\},
\end{aligned}
$$

where $L_{\mathrm{s}}$ is the effective mass of the soundboard, $C_{\mathrm{s}}$ is its effective compliance, $R_{\mathrm{S}}$ is its effective resistance, $L_{\mathrm{H}}$ is the mass of the hammer, $C_{\mathrm{f}}$ is the compliance of the hammer, $r_{0}$ is the characteristics impedance of the string, $i_{0}$ is the initial velocity of the hammer, and

$$
\left.\begin{array}{rl}
D= & \sqrt{\left\{\left(\alpha-\alpha_{\mathrm{s}}\right)^{2}+\left(\omega+\omega_{\mathrm{s}}\right)^{2}\right.} \\
& \times \sqrt{\left\{\left(\alpha-\alpha_{\mathrm{s}}\right)^{2}+\left(\omega-\omega_{\mathrm{s}}\right)^{2}\right\}} \\
\theta_{1}= & \sin ^{-1} \frac{\omega\left\{\left(\alpha^{2}-\alpha_{\mathrm{s}}^{2}\right)+\left(\omega^{2}-\omega_{\mathrm{s}}^{2}\right)\right\}}{\sqrt{\omega^{2}+\alpha^{2}} D}, \\
\theta_{2}= & -\sin ^{-1} \frac{\omega_{\mathrm{s}}\left\{\left(\alpha^{2}-\alpha_{\mathrm{s}}^{2}\right)+\left(\omega^{2}-\omega_{\mathrm{s}}^{2}\right)\right\}}{\sqrt{\omega_{\mathrm{s}}^{2}+\alpha_{\mathrm{s}}^{2}} D}
\end{array}\right\}
$$

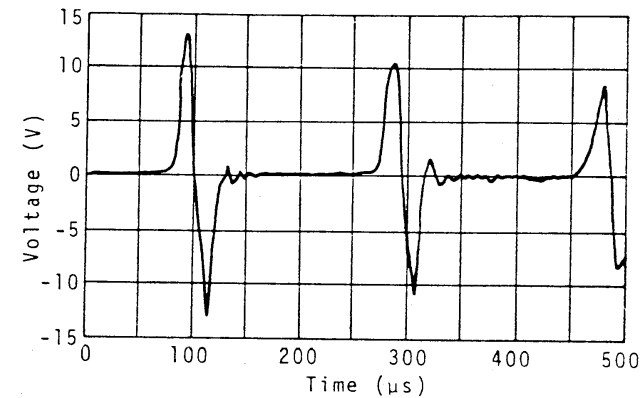

(b)

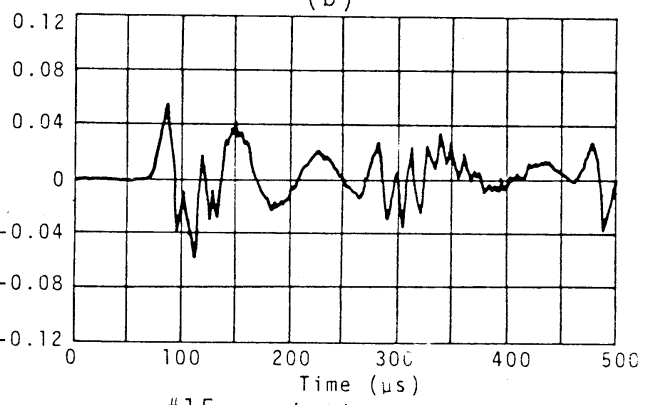

$\# 15$

$\left(b^{\prime}\right)$

$$
\begin{aligned}
& \alpha=\frac{1}{4 r_{0} C_{\mathrm{f}}}, \quad \alpha_{\mathrm{s}}=\frac{R_{\mathrm{s}}}{2 L_{\mathrm{s}}} \\
& \omega=\sqrt{\frac{1}{L_{\mathrm{H}} C_{\mathrm{f}}}-\frac{1}{16 r_{0}^{2} C_{\mathrm{f}}{ }^{2}}}, \\
& \omega_{\mathrm{s}}=\sqrt{\frac{1}{L_{\mathrm{s}} C_{\mathrm{s}}}-\frac{R_{\mathrm{s}}{ }^{2}}{4 L_{\mathrm{s}}}} .
\end{aligned}
$$

These equations represent a case where the system is driven by an external force of $P e^{-\alpha t} \sin \omega t$. To obtain equations for a case where the system is driven by a half-sine wave, the response to $P e^{-\alpha t}$. $\sin \omega(t-\pi / \omega) \mathbf{1}(t-\pi / \omega)$ should be added to them. Also a wave reflected from the fixed end of the string (which has a time lag of $2 a / c_{0}$ and a phase opposite to the original wave) must be added to them to obtain the vibration of the soundboard. The ratio of the 1st term (forced vibration) and the 2 nd term (free vibration) is determined by the relative magnitudes of $\sqrt{\omega^{2}+\alpha^{2}} / \omega$, and $\sqrt{\omega_{\mathrm{s}}{ }^{2}+\alpha_{\mathrm{s}}{ }^{2}} / \omega_{\mathrm{s}}$. Since numerical calculations for these case are complex even if a single resonance system is assumed, the use of a simulator is effective. An example for a case having two strings will be explained later. 


\section{CHARACTERISTICS OF PIANO SOUND}

Let us consider the characteristics of piano sound from the point of view of its generation. Assuming that a string-and-hammer system is linear, the contact force is represented by a half-sine wave approximately as shown in Fig. 7. A closer approximation than this can be given by taking account of the attenuation characteristic. ${ }^{1)}$ The hammer-string contact time $\tau_{0}$ is determined by the constants of the string and hammer. The maximum amplitude $A_{0}$ is proportional to the string striking-velocity. The change of tension of the string propagates along in two directions forming waves. The wave propagated towards the fixed end of the string reflects at the end, and the reflected wave propagates with a time lag of $2 a / c_{0}=T_{0} / \mathrm{m}$ and in the opposite phase, where $T_{0}$ is a period and $m$ is a struck string ratio. The wave also reflects at the bridge, and the waves overlap along the string. The vertical component of the tension ('vertical force') drives the bridge to vibrate the soundboard producing sound. When a sound spectrum at an initial stage is considered, the soundboard can be regarded as a fixed body, since its impedance is far greater than that of a string. The vertical force of the string in the first cycle can be expanded in a Fourier series as

$$
\begin{gathered}
A_{n}=-\frac{8}{\pi} \tau_{0} f_{0} A_{0} \frac{\cos \left(n \pi \tau_{0} f_{0}\right) \sin (n \pi / m)}{1-\left(2 n \tau_{0} f_{0}\right)^{2}} \\
\varphi_{n}=-n \pi\left(1+\tau_{0} f_{0}\right),
\end{gathered}
$$

where $A_{n}$ is the $n$-th harmonic of the amplitude, $\varphi_{n}$ is a phase of the $n$-th harmonic, and $f_{0}=1 / T_{0}$ is the fundamental frequency.

The higher the frequency of the vertical force, the

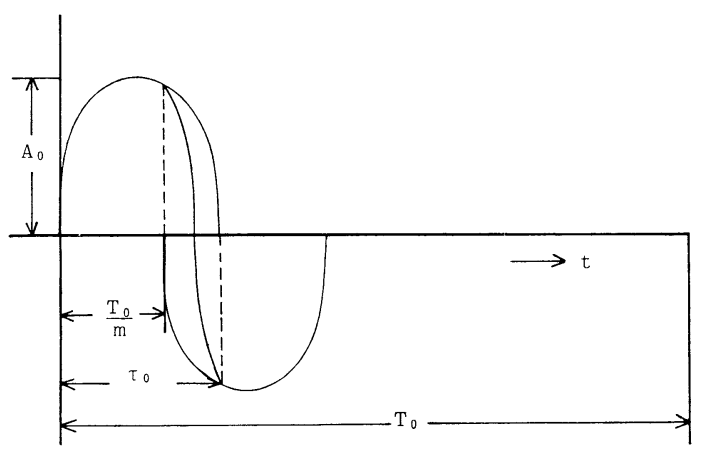

Fig. 7 Waveform of an ideal vertical force on a string. faster the propagation velocity along a string, since an actual string has both tension and elasticity. The frequency of each partial is represented by

$$
f_{n}=n f_{0}\left(1+B n^{2}\right)^{1 / 2} \fallingdotseq n f_{0}\left(1+\frac{B}{2} n^{2}\right),
$$

as in the previous paper, ${ }^{3)}$ where $B$ is a constant determined by the characteristic of a string.

Figure 8 shows the waveform of the vertical force of each $G$ string synthesized by using the abovedescribed procedure with constants for a middle class upright piano. The number of partials used for the simulation varies depending on its frequency, e.g. 30 for $\mathrm{G} 1$ and 6 for G6b. The waveform of a lower sound has a more pulse-like shape showing the effect of the dispersion due to the elasticity of its string, while the higher the frequency the closer to a sine wave.

The vertical force wave of a string and its reflected wave are superimposed at the bridge point, and this doubled force drives the soundboard. On the soundboard, both the forced vibration and a free vibration influenced by the driving-point impedance occur. Taking a case where the driving-point impedance has a single resonance system, let us compare the magnitudes of the 1 st and 2 nd terms of Eq. (2).

$$
\left.\begin{array}{l}
\text { 1st term: } 1 / \sqrt{1-\left(\frac{\sqrt{L_{\mathrm{H}} / C_{\mathrm{f}}}}{4 r_{0}}\right)^{2}} \\
\text { 2nd term : } 1 / \sqrt{1-\left(\frac{R_{\mathrm{s}}}{2 \sqrt{L_{\mathrm{s}} / C_{\mathrm{s}}}}\right)^{2}} .
\end{array}\right\}
$$
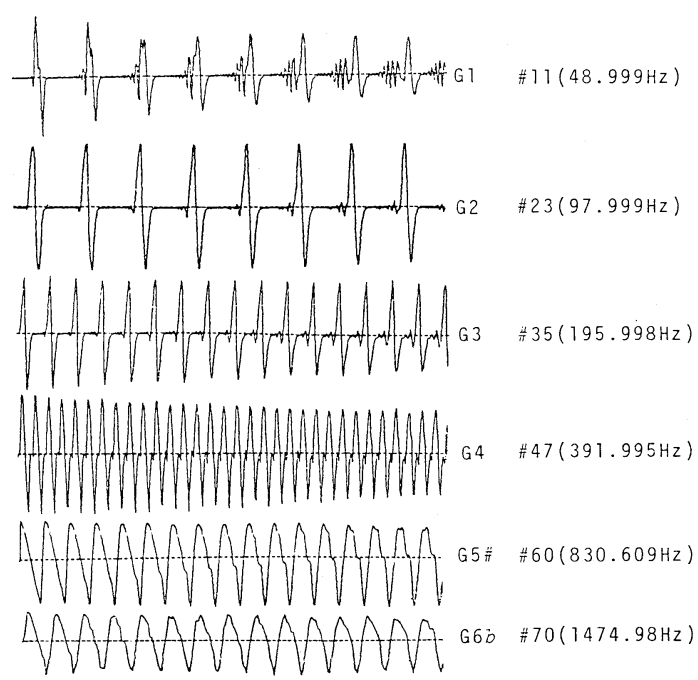

Fig. 8 Waveforms of vertical force obtained by spectrum synthesis. 


\section{NAKAMURA : CHARACTERISTICS OF PIANO SOUND}

The 1 st term is determined by the constants of a hammer and a string. The 2 nd term is determined by the constants of the soundboard. The ratio of the magnitudes of the forced vibration and the free vibration is related to the ratio of the impedance of the hammer system, and that of the soundboard system. Therefore, the characteristics of the soundboard, i.e. the timbre of a piano, is influenced by a matching of constants of the hammers, strings and the soundboard.

Vibration of a string attenuates due to its friction in the air and its acoustic radiation. The attenuation constant $\alpha$ is given by

$$
\alpha \propto G+A \omega^{2},
$$

as shown in the previous paper. ${ }^{3)}$ The attenuation due to the air is uniform independently of the frequency of vibration of the string. The attenuation due to the acoustic radiation is faster at a higher frequency. The attenuation due to the soundboard is complex. At the earlier stage of vibration, the attenuation is faster, forming a prompt sound. This is due to the fact that more than one string vibrate in phase. After this stage, the attenuation becomes slower, forming a aftersound. This is caused by difference in phases of strings with slightly different frequencies.

Figure 9 shows the waveforms of vibration of double string for key number 15 obtained by a digital simulator for $2 \mathrm{~s}$; (a) being the waveform of the velocity of vibration of the soundboard, and (b) is the waveform of the driving force of one of the two strings (the other string has a similar waveform to the one shown). The simulator has 80 meshes for each string. The two strings were tuned with a frequency difference of 2 cent. The soundboard was simulated by a single resonance system. The waveforms were sampled at $20 \mathrm{kHz}$.

In the waveform in Fig. 9(a), both a free vibration and a forced vibration influenced by the dispersion can be seen. The waveform has a fast attenuation in the first one second, and more gradual attenuation later, since the difference of the tuned frequency of the two string is only 2 cent. This trend cannot be seen in the waveform of the driving force in Fig. 9(b), but the effect of the free vibration of the soundboard appears in the waveform of the driving force at a later stage of the vibration.

Figure 10 shows the spectra of the abovedescribed vibrations. The spectra of the string

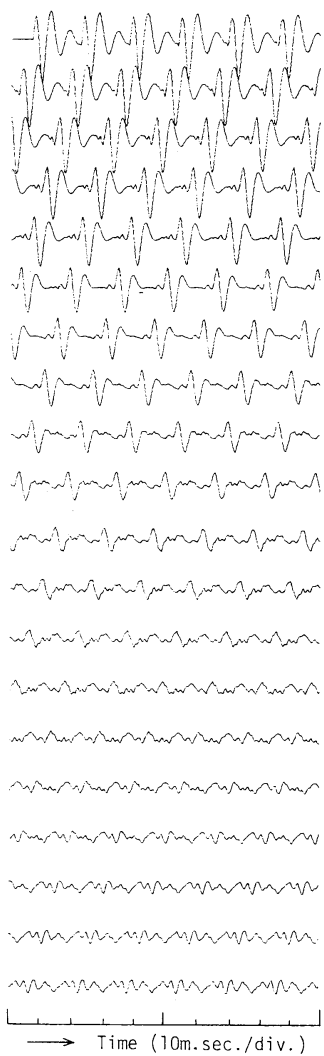

(a)

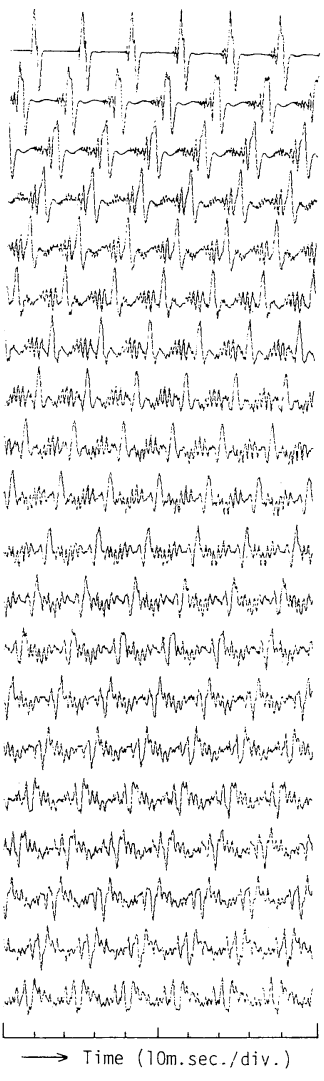

(b)
Fig. 9 Waveforms of vibrations of soundboard and string (key No. 15), obtained by digital simulation. (a): Velocity of vibration of soundboard at driving point. (b): Driving force on one of the strings.

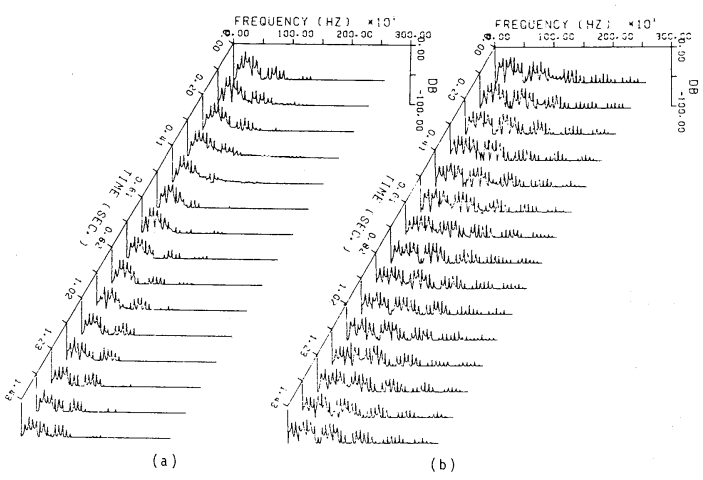

Fig. 10 Spectra of vibrations of soundboard and string (key No. 15), obtained by simulated waveforms. (a): Velocity of vibration of soundboard at driving point. (b) : Driving force on one of the strings. 


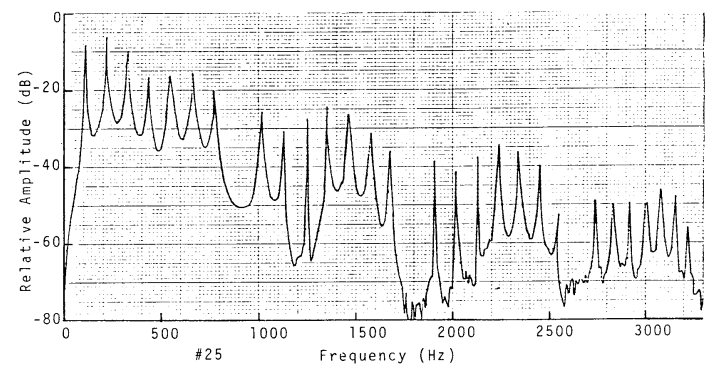

Fig. 11 Spectrum of velocity of vibration of soundboard, obtained by digital simulation.

vibration has partials over a very high frequency range. These partials are suppressed on the soundboard due to its resonance system. This makes the piano sound clear. Although actual piano sound produced by a soundboard having more than one resonance system will be more complex, the analysis shown above demonstrates the point.

The 8th partial and partials, having frequencies which are multiples of this frequency, can hardly be seen in Fig. 10, since the string striking point was chosen to be $1 / 8$ of the length of the string.

The high-frequency characteristic of an actual piano sound should be better than this simulation, due to the transmission characteristics of the actual soundboard, including its sound radiation characteristic.

Figure 11 shows a spectrum of the velocity of the soundboard vibration, at key number 25 (A2, 110 $\mathrm{Hz}$ ), which is assumed to be resistive and so obtained by a simulation. The simulation represents the vibration of two strings which differ by 2 cent in their frequencies. The simulation has 40 meshes for each string. The spectrum is calculated from a waveform over a period of about $0.1 \mathrm{~s}$. The higher partials are shifted more than multiples of the fundamental frequency.

The characteristics of piano sound can be summarized: - Main partials are formed by strings struck with a hammer, and their patterns modified by the driving impedance of the soundboard. 'A piano-like sound' is created by these partials and amplitude-decaying characteristics with the rapid decay of higher harmonics. In other words, if either the above-mentioned spectrum structure or the amplitude decaying characteristic is not in a certain range, it would be different from the piano-like sound.

As H. Fletcher ${ }^{7}$ has been piointed out, a slight inharmonicity makes the piano sound warmer. If each note has more than one string, it is possible to adjust the prompt sound, aftersound and partials by tuning each string, so that the piano sound becomes richer.

\section{CONCLUSION}

Following the process of generation of piano sound, its characteristics have been investigated so that the roles of sound spectra, attenuation characteristic, inharmonicity and beats in the piano timbre are clarified. It has been found that a matching of the hammers and strings on the soundboard is important, and that the driving-point impedance on the soundboard acts as a band-pass filter.

\section{ACKNOWLEDGEMENTS}

The author wishes to express his thanks to many people for their co-operation at various stages of this work which took a considerable time, and to Dr. Sizuo Ishiguro in England for his help in preparing the English version of this paper.

\section{REFERENCES}

1) I. Nakamura, "The vibration of a struck string (Acoustical research on the piano, Part 1), J. Acoust. Soc. Jpn. (J) 36, 504-512 (1980) (in Japanese); J. Acoust. Soc. Jpn. (E) 13, 311-321 (1992).

2) I. Nakamura, "Vibrational and acoustic characteristics of soundboard (Acoustical research on the piano, Part 3)," J. Acoust. Soc. Jpn. (J) 37, 510-517 (1981) (in Japanese); J. Acoust. Soc. Jpn. (E) 14, 429-439 (1993).

3) I. Nakamura, "Simulation of sound production mechanism (Acoustical research on the piano, Part 2)," J. Acoust. Soc. Jpn. (J) 37, 65-75 (1981) (in Japanese); J. Acoust. Soc. Jpn. (E) 14, 73-84 (1993).

4) J.P. Quitter, "Research and development on the piano," IRE Trans. AU-6, 96-103 (1956).

5) P. R. Dijksterhuis and T. Verhey, "An electronic piano," J. Audio Eng. Am. 17, 266-271 (1969).

6) O. H. Schuck and R. W. Young, "Observations on the Vibrations of piano strings," J. Acoust. Soc. Am. 15, 1-11 (1943).

7) H. Fletcher, E. D. Blackham, and R. Stratton, "Quality of piano tones," J. Acoust. Soc. Am. 34, 749-761 (1962).

8) D. W. Martin, "Decay rates of piano tones," J. Acoust. Soc. Am. 19, 535-541 (1947).

9) G. Weinreich, "Coupled piano strings," J. Acoust. Soc. Am. 62, 1474-1484 (1977).

10) T. C. Hundley, H. Benioff, and D. W. Martin, "Factors contributing to the multiple rate of piano tone 


\section{NAKAMURA : CHARACTERISTICS OF PIANO SOUND}

decay," J. Acoust. Soc. Am. 64, 1303-1309 (1978).

11) R. D. Weyer, "Time-frequency-structures in the attack transients of piano and harpsichord sounds. I," Acustica 35, 232-252 (1976).

12) I. Nakamura, M. Ohtsubaki, and T. Murai, "The vibration of a soundboard by a struck string-Consideration using an electronic simulator," Proc. Spring Meet. Acoust. Soc. Jpn., 189-190 (1968) (in Japanese). 